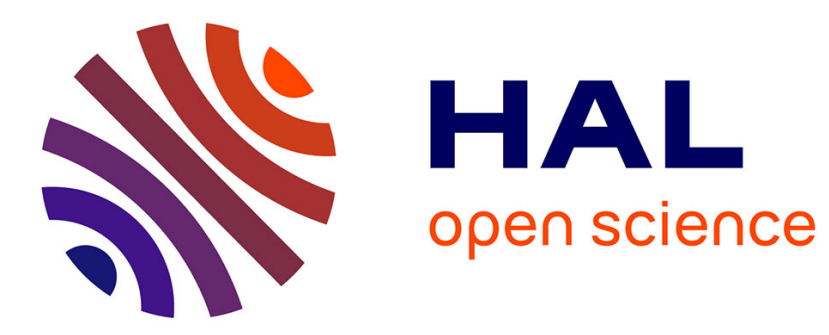

\title{
Adaptation de l'index francais de sélection laitière (INEL) au contexte des quotas
}

\author{
J.J. Colleau, Non Renseigné, P.L. Gastinel
}

\section{To cite this version:}

J.J. Colleau, Non Renseigné, P.L. Gastinel. Adaptation de l'index francais de sélection laitière (INEL) au contexte des quotas. Productions Animales, 1994, 7 (3), pp.151-167. hal-00896083

\section{HAL Id: hal-00896083 \\ https://hal.science/hal-00896083}

Submitted on 1 Jan 1994

HAL is a multi-disciplinary open access archive for the deposit and dissemination of scientific research documents, whether they are published or not. The documents may come from teaching and research institutions in France or abroad, or from public or private research centers.
L'archive ouverte pluridisciplinaire HAL, est destinée au dépôt et à la diffusion de documents scientifiques de niveau recherche, publiés ou non, émanant des établissements d'enseignement et de recherche français ou étrangers, des laboratoires publics ou privés. 
INRA Prod. Anim., 1994, 7 (3), 151 - 167.
J.J. COLLEAU, D. REGALDO*, P.L. GASTINEL

INRA Station de Génétique Quantitative et Appliquée 78352 Jouy-en-Josas Cedex

* Institut de l'Elevage, Département de Génétique et de Contrôle des performances 149, Rue de Bercy 75595 Paris Cedex 12

\section{Adaptation de l'index francais de sélection laitière (INEL) au contexte des quotas}

L'évolution du contexte économique amène régulièrement le généticien à s'interroger sur le bien-fondé des orientations globales de la sélection qu'il opère ou recommande. A cet égard, l'instauration en 1984 de quotas de production laitière au sein de la Communauté Economique Européenne est un exemple particulièrement spectaculaire de modification de contexte économique. Le réajustement de l'index de synthèse économique laitière, appliqué à l'ensemble des races bovines laitières françaises, constitue une réponse possible à ce genre de situation.

D'une manière générale, l'index d'un reproducteur est l'estimée de sa valeur génétique compte tenu des informations dont on dispose. L'index peut concerner un caractère particulier ou une combinaison de plusieurs caractères. C'est dans ce dernier cas qu'on parle d'index de synthèse.

L'objectif principal de cet article est de présenter l'argumentation technique utilisée

\section{Résumé}

La sélection des races bovines laitières s'effectue en France au travers d'un index de synthèse économique laitière (INEL). La formulation de celui-ci a été remise à jour en 1993 pour mieux tenir compte de la situation concrète des quotas laitiers, avec modulation pour les dépassements de taux butyreux par rapport à la référence.

L'article explicite les raisonnements, hypothèses et paramètres tant économiques que génétiques, utilisés pour l'examen de plusieurs alternatives possibles. Les hypothèses essentielles sont la constance des surfaces et des quotas par exploitation ainsi que la prise en considération de marges nettes incluant les charges fixes.

La solution finalement retenue (INEL 1993) n'accorde plus aucun intérêt économique à l'augmentation de la production de matière grasse par vache. La sélection sur ce nouvel index entraînera une augmentation du taux protéique et une amélioration du rapport taux protéique/taux butyreux. Le taux butyreux diminuera en race Holstein et restera approximativement constant dans les races Normande et Montbéliarde. pour l'élaboration de la dernière version de l'index de synthèse économique laitière (INEL) et d'exposer les réponses attendues à la sélection sur ce nouvel index. En exposé préliminaire, de manière à faciliter la compréhension d'un domaine qui n'est peut-être pas familier à nombre de lecteurs, on rappellera la signification de concepts spécifiques qui se situent à l'interface de la génétique quantitative et de l'économie, notamment ceux de pondérations économiques et d'objectifs de sélection. A la suite de l'exposé principal qui consistera à présenter les résultats de simulation, on discutera la solution retenue et on la comparera aux travaux analogues réalisés à l'étranger.

\section{1 / Notions théoriques et formalisation du problème}

\subsection{Objectif de sélection}

Imaginons que l'on connaisse les valeurs génétiques vraies $g_{1}, g_{2}, \ldots, g_{n}$ d'un reproducteur pour $n$ caractères d'importance économique. Ce que l'on appelle objectif de sélection est tout simplement une fonction $\mathrm{H}$ ( $\mathrm{g}_{1}$, $\left.\mathrm{g}_{2}, \ldots, \mathrm{g}_{\mathrm{n}}\right)$ qui donne de façon tout à fait expli- 
cite, par une formulation mathématique qui dépend du problème posé, un seul résultat : la valeur économique de synthèse de ce reproducteur. L'intérêt de définir une telle fonction est de maximiser l'efficacité économique de la sélection. Si l'on choisit une proportion donnée des animaux (par exemple 10\%) pour en faire des reproducteurs utilisés à large échelle, la valeur économique globale de leur descendance est maximale si ces reproducteurs sont triés sur $H$. Ceci est plus efficace en particulier que la sélection avec seuils éliminatoires intuitifs pour $g_{1}, \ldots ., g_{n}$. Si la formulation de $\mathrm{H}$ est correcte, on comprend bien qu'on puisse aboutir à un tel résultat. Il est d'ailleurs démontré de manière théorique dans les ouvrages spécialisés de génétique quantitative (Ollivier 1981, Minvielle 1990).

La fonction $\mathrm{H}$ peut être complexe. C'est le cas si les caractères ne s'expriment pas au même moment dans la vie de l'animal et avec la même fréquence (par exemple, une vache a l'occasion d'extérioriser son génotype laitier pendant trois lactations en moyenne, alors que son génotype boucher n'est exprimé qu'une fois, à la réforme). Par ailleurs, l'impact économique d'une variation donnée d'un $g_{i}$ particulier peut ne pas être constant : par exemple, l'incidence d'une diminution de la fertilité est d'autant plus marquée que celle-ci est faible au départ, comme l'a montré Boichard (1990).

Le cas le plus complexe est celui où tous les phénomènes précédents coexistent. Elsen et al (1986) discutent les difficultés rencontrées et les solutions proposées dans la littérature scientifique pour de tels cas.

Le cas le plus simple est celui où les caractères s'expriment simultanément et où la fonction $\mathrm{H}$ est de type linéaire, au moins pour des variations modérées autour de moyennes (linéarisation de fonctions complexes de plusieurs variables par un développement limité de Taylor autour des moyennes). Cette situation se rencontre précisément pour les caractères de production laitière et de richesse du lait. On a alors :

$\mathrm{H}=\mathrm{k}\left(\mathrm{m}_{1} \mathrm{~g}_{1}+\mathrm{m}_{2} \mathrm{~g}_{2}+\ldots \mathrm{m}_{\mathrm{n}} \mathrm{g}_{\mathrm{n}}\right)$

où les $\mathrm{m}_{\mathrm{i}}$ correspondent aux marges unitaires de chacun des caractères 1 à $n$ et où $k$ est une constante d'échelle (nombre de lactations ou nombre de descendants suivant les cas). Il est à noter que le résultat du tri des reproducteurs, et donc les réponses pour chaque caractère, ne dépend pas de $\mathrm{k}$. Si l'on s'intéresse uniquement à ces aspects, on peut prendre $\mathrm{k}=1$ pour simplifier.

Tous les développements précédents ne prennent pas en compte des variations possibles dans le temps de la forme même de la fonction $\mathrm{H}$, liée notamment aux fluctuations à long terme des conditions économiques (par exemple instauration de quotas puis suppression mais avec non garantie du prix du lait). La sélection doit donc s'effectuer après des réexamens périodiques du type de pondérations à utiliser.

\section{2 / Modalités de calcul des pondérations de l'objectif de sélection}

\section{a / pondérations sans contrainte}

Les $\mathrm{m}_{\mathrm{i}}$ correspondent alors à la différence entre le supplément de recettes et de coûts, liés à une augmentation d'une unité de $g_{j}$. On peut essayer d'évaluer ces paramètres à partir de l'analyse statistique de données réelles. Cependant, cette méthode présente le risque de donner des résultats biaisés par l'effet conjoint de variables parasites non prises en compte dans l'analyse (Elsen et al 1986). C'est la raison pour laquelle il est préférable, ce qui ne veut pas toujours dire aisé, de modéliser le fonctionnement des unités de production (modèle microéconomique) ou de filières entières (modèle macroéconomique), suivant la perspective choisie.

\section{b / pondérations sous contrainte de quotas}

Cette contrainte introduit des complications, car une variation de niveau génétique pour le caractère soumis à quota a non seulement pour conséquence de faire varier le bilan économique au niveau de chaque animal mais aussi de faire varier le nombre d'animaux. On comprend ainsi très facilement que si le bilan d'une exploitation ne dépend que d'un caractère (par exemple la quantité de lait), l'instauration d'un quota pour ce caractère, en situation de progrès génétique, aura pour effet de diminuer le nombre total d'animaux exprimant ce progrès génétique. La nouvelle marge par unité de caractère, rapportée à chaque animal, peut être alors différente et même inférieure à l'ancienne marge. Si le bilan économique de l'exploitation dépend d'un deuxième caractère et si celui-ci n'est pas soumis à quota, la marge générée par un progrès génétique pour ce caractère n'est pas modifiée puisque ce progrès ne contribue pas alors à la réduction des effectifs.

Pour calculer les nouvelles marges, il est alors nécessaire de formaliser à l'aide de paramètres et d'équations adéquats, le bilan économique d'une exploitation et de calculer à partir de ce modèle l'effet net d'une variation unitaire pour le caractère soumis à quota. Le principe de ce calcul est immédiat puisqu'il ne nécessite que le calcul de dérivées algébriques concernant des fonctions en général très simples.

\section{c / contraintes simultanées de quota de production et de surface d'exploitation}

Si l'on introduit une contrainte de surface, on est alors obligé d'intégrer dans le bilan les variations d'activité directement introduites par l'amélioration génétique. Si la superficie consacrée aux bovins laitiers diminue, notamment à cause d'une réduction des effectifs, la superficie alors ouverte à des activités de substitution (cultures, autres formes d'élevage) doit être prise en considération. 


\section{d / nécessité et forme d'une solution générale}

Compte tenu de ces deux types de contrainte et compte tenu du fait que la contrainte de production peut concerner non un caractère mais une combinaison de caractères, il est alors nécessaire de chercher à établir une formulation générale qui permette d'analyser une série de situations diverses.

Cette formulation figure en annexe. Elle permet notamment d'analyser l'incidence de quotas différents : production laitière, quantité de matière grasse ou production laitière modulée en fonction du taux de matière grasse.

On voit que dans la situation c (quota de production et surface d'exploitation constants), la nouvelle marge correspondant à une augmentation d'une unité pour un caractère donné est égale à l'ancienne marge modifiée par deux termes correctifs.

Le premier terme correctif correspond à l'incidence de cette augmentation sur la surface utilisée par animal. Le second correspond à l'incidence de cette augmentation sur la marge totale par le biais d'une variation de l'effectif des animaux.

Cette formulation permet en particulier d'évaluer quantitativement le bilan net d'une situation conflictuelle où l'amélioration génétique permet d'obtenir des animaux de plus en plus performants au niveau individuel mais en effectif de plus en plus limité, contraignant l'éleveur à se tourner de plus en plus vers des activités moins rentables que la production laitière.

\section{3 / Index synthétique de sélection}

Une fois établie la fonction $\mathrm{H}=\Sigma \mathrm{m}_{\mathrm{i}} \mathrm{g}_{\mathrm{i}}$, il faut alors sélectionner les reproducteurs sur un index synthétique I qui doit être en corrélation maximum avec $\mathrm{H}$ (Hazel 1943, Ollivier 1981, Minvielle 1990).

Si l'on connaît toutes les relations statistiques (héritabilités et corrélations génétiques notamment) concernant les caractères de base, il est alors possible d'estimer conjointement les $\mathrm{n}$ valeurs génétiques $\mathrm{g}_{1}, \ldots \mathrm{g}_{n}$ au travers de $\mathrm{n}$ index élémentaires $\mathrm{I}_{1}, \ldots, \mathrm{I}_{\mathrm{n}}$ (Indexation multivariate). La forme de la solution est alors très simple

$$
\mathrm{I}=\Sigma \mathrm{m}_{\mathbf{i}} \mathrm{I}_{\mathbf{i}}
$$

Si les $I_{i}$ sont obtenus grâce à des indexations séparées (et non conjointes), la formulation précédente n'est plus valable à moins qu'on ne soit dans le cas très spécial où il n'existe aucune relation entre caractères, tant génétique que non génétique.

\section{4 / Réponses à la sélection}

Si l'on connaît les paramètres statistiques qui ont permis le calcul des index et si l'on connaît la précision de ceux-ci en prenant en considération les effectifs d'animaux impliqués et leur apparentement, il est alors possible d'évaluer a priori l'effet d'une variation unitaire de I sur les niveaux génétiques $g_{1} \ldots g_{n}$ de chacun des caractères, et donc sur $\mathrm{H}$.

La pression de sélection exercée sur les reproducteurs permet en outre de moduler les variations enregistrées sur I.

On voit donc qu'il est possible de prédire le résultat (réponse à la sélection) d'une politique de sélection caractérisée par son degré de sévérité et par le type d'index synthétique utilisé (Ollivier 1981, Minvielle 1990).

\section{2 / Lignes directrices de l'application à l'INEL}

En raison du sujet abordé, l'amélioration génétique, et des durées très importantes séparant décisions de sélection et impact réel à grande échelle dans la population (supérieures à 10 ans quand on raisonne au niveau des taureaux d'insémination), on s'est efforcé d'imaginer les conséquences assez lointaines de décisions de sélection actuelles, en dépit des incertitudes sur la conjoncture économique future.

\section{Les hypothèses sont les suivantes :}

1- Les quotas et surfaces pour chaque exploitation laitière ne varient pas. Cette hypothèse volontairement très sévère par rapport à la réalité présente permet d'apprécier le revenu réel lié à la sélection, en s'affranchissant d'une autre hypothèse encore plus forte pour l'avenir, et peu souhaitable sociologiquement : celle d'une diminution permanente et acceptée du nombre de producteurs laitiers.

2- Le bilan économique de l'exploitation est évalué au travers de la marge nette, hors charges de travail : il permet précisément d'apprécier comment le travail peut être effectivement rémunéré.

3- On considère que sur le long terme, les charges fixes tendent à s'ajuster au nombre de vaches et à la surface affectée aux activités de substitution. Cette hypothèse représente une différence fondamentale par rapport aux raisonnements à court terme, relatifs par exemple à l'effet du mode de conduite d'un troupeau laitier. Les charges fixes d'amortissement de matériel et de bâtiment sont invariables sur le court terme. Sur le long terme (10 à $20 \mathrm{ans}$ ), on considère que ces matériels et bâtiments sont renouvelés et dans ce cas adaptés à la structure du cheptel et de l'exploitation.

4- Sur le long terme, les charges fixes bovines sont proportionnelles au nombre de vaches. L'incidence possible des variations de niveau de production par vache sur les charges fixes par vache est considérée comme nulle.

5- Les besoins alimentaires, en unités d'énergie, correspondant à la production laitière sont proportionnels à cette production ou, ce qui est équivalent, les besoins par $\mathrm{kg}$ de lait supplémentaire sont constants. 


\section{3 / Critères économiques détaillés, sources et paramétrage}

Les paramètres technico-économiques nécessaires ont été déterminés en trois étapes. D'abord les situations-types ont été définies par les Etablissements Départementaux de l'Elevage de Bretagne. L'Institut de l'Elevage a ensuite procédé à l'estimation de marges brutes ramenées à la vache laitière compte tenu des connaissances préalables détaillées d'exploitations laitières spécialisées de Bretagne. Enfin, une fourchette de charges fixes ramenées à la vache laitière ou à l'hectare de l'activité de substitution a été estimée par l'Institut de l'Elevage d'après les cas-types décrits par le réseau Eleveurs de Bovins Demain.

\section{1 / Les situations-types}

Les situations-types (tableau 1) font varier : le paiement du gramme différentiel de taux protéique,TP, $(3,5$ centimes correspond approximativement à la situation actuelle et 5 centimes à une hypothèse haute) ; le niveau de production laitière à $5000 \mathrm{~kg}, 7000 \mathrm{~kg}$ et $9000 \mathrm{~kg}$ avec deux niveaux de taux butyreux (TB) à $41 \%$ et $38 \%$; le coût de l'UF lait concentré avec deux modalités à $1,35 \mathrm{~F}$ et à $1 \mathrm{~F}$ et le type d'exploitation selon deux variantes (EBD 7 et EBD 2) rencontrées en BretagnePays de Loire (Anonyme 1987).

De plus, pour la production d'un kg de lait supplémentaire, les besoins en UFL - soit 0,45 ou $0,55 \mathrm{UFL} / \mathrm{kg}$ (valeur volontairement pessi- miste) - sont couverts par le concentré dans les proportions $1 / 2$ ou $2 / 3$ alors que le taux moyen de couverture par le concentré, au niveau de l'ensemble de la production initiale, est de l'ordre de $1 / 3$. On tient donc compte du fait que l'augmentation de la production coûte cher en concentré. Ces besoins sont imputés à la matière grasse, à la matière protéique et au vecteur (lait hors matière grasse et matière protéique) dans les proportions respectives de $55 \%, 22,5 \%, 22,5 \%$ (Wilmink 1988, Vermorel 1991, communication personnelle).

Les situations-types sont étudiées dans trois contextes : quota matière grasse, quota lait et absence de quota.

\section{2 / Les marges brutes}

Les produits considérés proviennent du lait vendu, des veaux de huit jours purs et croisés ainsi que des réformes.

Les charges opérationnelles incluent, pour la vache laitière et sa suite, l'alimentation (fourrages, concentrés, complément minéral et vitaminique, poudre de lait, aliment jeune bovin), les frais d'élevage, les frais vétérinaires, les taxes à la production, la paille et divers.

La marge brute liée à l'activité de substitution a été fixée à $2000 \mathrm{~F}$ ou $4000 \mathrm{~F}$ par hectare.

\section{3 / Les charges fixes}

Les comptes de gestion des fermes du réseau $\mathrm{EBD}$ permettent de connaître le niveau des dépenses de structure (charges sociales, fermage, taxes foncières, consommations d'énergie et d'eau, entretien mécanique), les

Tableau 1. Paramètres caractéristiques des situations étudiées.

\begin{tabular}{|c|c|c|c|c|c|c|}
\hline \multirow[t]{2}{*}{ Situation } & \multirow{2}{*}{$\begin{array}{l}\text { Production } \\
\text { laitière } \\
\text { kg }\end{array}$} & \multicolumn{3}{|c|}{ Paiement du lait } & \multicolumn{2}{|c|}{ Par kg } \\
\hline & & $\mathrm{F} / \mathrm{kg}$ & c/g TB & e/g TP & $\begin{array}{c}\text { Besoins } \\
\text { UF/kg } \\
\text { supplémentaire }\end{array}$ & $\begin{array}{c}\text { (\% couverture } \\
\text { par le concentré) }\end{array}$ \\
\hline $\mathrm{R}$ (Référence) & 7000 & 2 & 2 & 5 & 0,45 & $(50)$ \\
\hline $\mathbf{B}$ (Basse) & 5000 & . & $"$ & " & 0,55 & $"$ \\
\hline M (Moyenne) & 7000 & $"$ & $"$ & $"$ & $"$ & $"$ \\
\hline $\mathrm{H}$ (Haute) & 9000 & " & $"$ & " & $"$ & (67) \\
\hline $\begin{array}{l}\text { Ma (Moyenne } \\
\text { paiement actuel) }\end{array}$ & 7000 & " & $"$ & 3,5 & $"$ & $(50)$ \\
\hline
\end{tabular}

Variantes étudiées à l'intérieur des situations :

1- Marges nettes ( $\mathrm{F} / \mathrm{vache})$

$\begin{array}{lll}\mathrm{R}: 3700 / 4700 / 5700 & \text { B }: 2350 / 3350 & \mathrm{M}: 3700 / 4700 / 5700 \\ \mathrm{H}: 5800 / 6800 & \mathrm{Ma}: 3700 / 4700 / 5700 & \end{array}$

2- Marge nette de l'activité de substitution : $500 \mathrm{~F}$ ou $1500 \mathrm{~F} / \mathrm{ha}$

3- Répartition des besoins par kg de lait supplémentaire : Vecteur V $22.5 \%$; MG $55 \%$; MP $22.5 \%$

$(0,11 \mathrm{UF} / \mathrm{kg} \mathrm{V} \quad 6,51 \mathrm{UF} / \mathrm{kg} \mathrm{MG} \quad 3,16 \mathrm{UF} / \mathrm{kg} \mathrm{MP}$ pour $0,45 \mathrm{UF} / \mathrm{kg}$ lait)

ou $(0,13 \mathrm{UF} / \mathrm{kg} \mathrm{V} \quad 7,96 \mathrm{UF} / \mathrm{kg} \mathrm{MG} \quad 3,87 \mathrm{UF} / \mathrm{kg} \mathrm{MP}$ pour $0,55 \mathrm{UF} / \mathrm{kg}$ lait)

4- Référence EBD : (0,30 F/UF Fourrage $8500 \mathrm{UF} / \mathrm{ha}$ et 0,68 ha par VL et sa suite $)$

$(0,37 \mathrm{~F} / \mathrm{UF}$ Fourrage $\quad 6800 \mathrm{UF} / \mathrm{ha}$ et 0,80 ha par VL et sa suite)

5- TB : 41 ou $38 \mathrm{~g} / \mathrm{kg}, \mathrm{TP}: 31 \mathrm{~g} / \mathrm{kg}$

6- Concentré à $1,35 \mathrm{~F} / \mathrm{UF}$ ou $1 \mathrm{~F} / \mathrm{UF}$ 
amortissements et frais financiers liés d'une part au matériel et d'autre part aux bâtiments (18\% de la valeur d'inventaire dans les deux cas). Les charges de travail ne sont pas prises en considération.

Les charges de structure et d'amortissement du matériel ont été réparties entre l'atelier laitier et les autres activités au prorata de la surface alors que les charges d'amortissement de bâtiments ont été entièrement affectées aux productions animales. Quand un atelier laitier et un atelier "viande" coexistent, les charges fixes sont réparties au prorata des UGB impliquées dans ces deux activités.

D'après les 9 cas d'EBD analysés en Bretagne et Pays de Loire (Anonyme 1987), les charges fixes ramenées à la vache laitière sont estimées, après prise en considération de l'inflation, à $4600 \mathrm{~F}$ en 1993 , les extrêmes étant de $4100 \mathrm{~F}$ et de $5200 \mathrm{~F}$. Pour les 10 cas EBD analysés dans l'Est de la France (Anonyme 1991), les résultats homologues sont de $4900 \mathrm{~F}$ (avec des extrêmes de $4600 \mathrm{~F}$ et de $5200 \mathrm{~F}$ ) et les charges fixes pour la production végétale de substitution s'établissent à $3400 \mathrm{~F} /$ ha (extrêmes de $2500 \mathrm{~F}$ et $4900 \mathrm{~F}$ ).

En conséquence, on a retenu trois niveaux possibles de charges fixes à $4000,5000 \mathrm{ou}$ $6000 \mathrm{~F}$ par vache laitière, et 2500,3500 ou $4500 \mathrm{~F}$ par ha de substitution, en bornant la marge nette à 0 dans ce cas.

\section{4 / Pondérations économiques}

\section{1 / Caractères impliqués}

Il s'agit de la quantité de matière protéique (MP), de matière grasse (MG) et du vecteur (V), c'est-à-dire de la quantité de lait hors MP et hors $\mathrm{MG}$, toutes exprimées en $\mathrm{kg}$. Les pon- dérations correspondent à l'effet économique net d'une augmentation de $1 \mathrm{~kg}$ pour chacune des trois variables.

Rappelons que cet effet économique net se décompose en :

- variation de marge par vache quand le caractère progresse de $1 \mathrm{~kg}$. Elle seule intervient hors quota si l'évolution de l'atelier laitier n'affecte pas les autres activités.

- valorisation par les activités de substitution des variations de surface par vache. Elle intervient si l'atelier laitier s'ajuste au détriment des autres activités du fait d'une surface limitée.

- variation de marge due à la réduction des effectifs. Elle intervient si le caractère est contraint par le quota. C'est le cas de MG s'il y a quota MG ; du vecteur, de la MG et de la MP, s'il y a quota lait (sans ou avec correction pour les variations de TB). Elle dépend alors de la réduction d'effectif imposée par le quota de l'exploitation, et de l'écart de marge entre les activités laitières et de substitution.

Tableau 2. Valeur économique (variation de marge par $\mathrm{kg}$ supplémentaire) pour la matière grasse (MG), la matière protéique (MP) et le vecteur (V), c'est à dire la différence Lait - MG - MP, en l'absence de quota.

\begin{tabular}{|c|c|c|c|}
\hline \multirow{2}{*}{$\begin{array}{c}\text { Situation } \\
\text { (voir tableau 1) }\end{array}$} & \multicolumn{3}{|c|}{ Valeur économique (F/kg) } \\
\cline { 2 - 4 } & = Lait MG-MP & MG & MP \\
\hline R & $-0,45$ & 14,3 & 47,0 \\
B & $-0,47$ & 13,1 & 46,5 \\
M & & & \\
$\mathrm{H}$ & $-0,49$ & 11,7 & 45,8 \\
$\mathrm{Ma}$ & 0,01 & 13,6 & 31,9 \\
\hline
\end{tabular}

Tableau 3. Valeur économique (variation de marge par kg supplémentaire) pour la matière grasse (MG), la matière protéique (MP) et le vecteur $(V)$, c'est à dire la différence Lait - MG - MP, en situation de quota "matière grasse".

\begin{tabular}{|c|c|c|r|r|r|r|c|}
\hline \multirow{2}{*}{$\begin{array}{c}\text { Situation } \\
\text { (voir tableau 1) }\end{array}$} & Marge nette & \multicolumn{6}{|c|}{ Marge nette (F) / ha libéré } \\
\cline { 3 - 8 } & F/VL & V & MG & MP & V & MG & MP \\
\hline \multirow{2}{*}{ R } & 3700 & & 2,3 & & & 4,3 & \\
& 4700 & $-0,45$ & $-1,2$ & 46,9 & $-0,46$ & 0,8 & 46,8 \\
& 5700 & & $-4,6$ & & & $-2,7$ & \\
B & 2350 & $-0,47$ & 3,1 & 46,3 & $-0,48$ & 5,9 & 46,1 \\
& 3350 & & $-1,8$ & & & 1,1 & \\
M & 3700 & & 1,1 & & & 3,0 & \\
& 4700 & $-0,47$ & $-2,4$ & 46,3 & $-0,48$ & $-0,5$ & 46,1 \\
& 5700 & & $-5,9$ & & & $-4,0$ & \\
H & 5800 & $-0,50$ & 3,3 & 45,7 & $-0,50$ & $-1,8$ & 45,5 \\
& 6800 & & $-6,0$ & & & 4,5 & \\
Ma & 3700 & & 1,6 & & & 3,5 & \\
& 4700 & 0,01 & $-1,9$ & 31,8 & 0 & 0 & 31,6 \\
& 5700 & & $-5,4$ & & & $-3,5$ & \\
\hline
\end{tabular}


Tableau 4. Valeur économique (variation de marge par $\mathrm{kg}$ supplémentaires) pour la matière grasse (MG), la matière protéique $(M P)$ et le vecteur $(V)$, c'est à dire la différence Lait - MG - MP, en situation de quota "lait".

\begin{tabular}{|c|c|c|c|c|c|c|c|}
\hline \multirow{3}{*}{$\begin{array}{c}\text { Situation } \\
\text { (voir tableau 1) }\end{array}$} & \multirow{3}{*}{$\begin{array}{c}\text { Marge nette } \\
\text { F/VL }\end{array}$} & \multicolumn{6}{|c|}{ Marge nette (F) / ha libéré } \\
\hline & & \multicolumn{3}{|c|}{500} & \multicolumn{3}{|c|}{1500} \\
\hline & & V & MG & MP & V & MG & MP \\
\hline $\mathrm{R}$ & $\begin{array}{l}3700 \\
4700 \\
5700\end{array}$ & $\begin{array}{l}-0,93 \\
-1,08 \\
-1,22\end{array}$ & $13,3-13,6$ & $46,2-46,5$ & $\begin{array}{l}-0,84 \\
-0,99 \\
-1,13\end{array}$ & $13,0-13,3$ & $46,1-46,4$ \\
\hline B & $\begin{array}{l}2350 \\
3350\end{array}$ & $\begin{array}{l}-0,87 \\
-1,07\end{array}$ & & & $\begin{array}{l}-0,75 \\
-0,95\end{array}$ & & \\
\hline M & $\begin{array}{l}3700 \\
4700 \\
5700\end{array}$ & $\begin{array}{l}-0,96 \\
-1,10 \\
-1,24\end{array}$ & $12,1-12,4$ & $45,6-45,9$ & $\begin{array}{l}-0,87 \\
-1,01 \\
-1,15\end{array}$ & $11,7-12,1$ & $45,4-45,8$ \\
\hline $\mathrm{H}$ & $\begin{array}{l}5800 \\
6800\end{array}$ & $\begin{array}{l}-1,10 \\
-1,21\end{array}$ & $10,8-10,9$ & $45,0-45,1$ & $\begin{array}{l}-1,03 \\
-1,14\end{array}$ & $10,6-10,7$ & $44,9-45,0$ \\
\hline $\mathrm{Ma}$ & $\begin{array}{l}3700 \\
4700 \\
5700\end{array}$ & $\begin{array}{l}-0,48 \\
-0,62 \\
-0,76\end{array}$ & $12,6-12,8$ & $31,1-31,3$ & $\begin{array}{l}-0,39 \\
-0,53 \\
-0,67\end{array}$ & $12,2-12,5$ & $30,9-31,2$ \\
\hline
\end{tabular}

\section{2 / Absence de quotas}

En l'absence de quotas (tableau 2), la valeur économique correspond à la différence entre le paiement et le coût alimentaire marginal de production d'un $\mathrm{kg}$ supplémentaire. Elle est très proche de la recette correspondante, sauf pour la MG qui absorbe $55 \%$ de l'énergie nécessaire pour produire un $\mathrm{kg}$ de lait en plus. La modalité de paiement choisie ( $2 \mathrm{~F} / \mathrm{l}, 2 \mathrm{c} / \mathrm{g} \mathrm{TB}$, $5 \mathrm{c} / \mathrm{gTP})$ pousse à réduire le vecteur, à rechercher la MG et davantage encore la MP.

\section{3 / Quota MG}

Le quota MG n'affecte que la valeur économique de la MG (tableau 3), seul caractère susceptible de modifier l'effectif du troupeau. Cette valeur économique devient alors beaucoup plus faible, voire négative. On constate que cette dernière situation survient quand la marge nette par vache est élevée et (ou) quand la marge nette de substitution est faible.

En raison du sens que nous donnons au terme "valeur économique", ceci veut dire très exactement que l'éleveur a très peu ou pas du tout intérêt à augmenter la production de matière grasse par vache.

\section{4 / Quota lait}

Tout changement concernant le vecteur, la MG ou la MP modifie l'effectif du troupeau. Cependant la contribution de la MG ou de la MP au volume total est faible, c'est pourquoi c'est essentiellement le vecteur $V$ qui est soumis à quota et qui voit sa valeur économique chuter le plus (de 100\%, ordre de grandeur). Les réductions de valeur économique pour MG et MP sont beaucoup plus faibles : 2 à $8 \%$. Ce type de quota pousse donc à la recherche de taux élevés (tableau 4).
Le contexte "quota lait" est aussi celui où la variabilité des objectifs entre élevages est la plus marquée, rendant ainsi plus délicate l'orientation générale des schémas.

\section{5 / Coefficients des index synthétiques}

En raison de la formulation de lindex INEL précédent (INEL 1989), on a choisi d'exprimer ces index en fonction de la matière protéique, de la matière grasse et du taux protéique. Les techniques de régression multiple, connaissant tous les paramètres génétiques impliqués, permettent d'obtenir les coefficients adéquats, même si la fonction économique $\mathrm{H}$ ne porte pas directement sur le taux protéique mais sur le vecteur. Par ailleurs, pour limiter le nombre de coefficients à mémoriser, nous avons choisi d'affecter, par convention, un poids 1 à la matière protéique. On sait en effet que la modulation d'effets d'échelle ne modifie pas les résultats de la sélection. L'index synthétique est alors de la forme:

$\mathrm{MP}+\mathrm{a} \mathrm{MG}+\mathrm{b} \mathrm{TP}$

(kg) $\quad(\mathrm{kg}) \quad(\mathrm{g} / \mathrm{kg})$

Il doit être rappelé ici que les coefficient a et $b$ procèdent d'une synthèse entre des considérations économiques (pondérations économiques) et des considérations statistiques (connaissance de la variabilité génétique des caractères considérés et de leurs liaisons).

En l'absence de quota, a est de l'ordre de 0,4 et $\mathrm{b}$ de l'ordre de 1,5, quand l'index de sélection est celui d'un taureau évalué sur 50 filles. En situation de quota matière grasse, l'index optimal est approximativement MP $+2 \mathrm{TP}$ (tableau 5). En situation de quota lait, l'index optimal est en moyenne de MP + MG + $10 \mathrm{TP}$, en notant une extrême variabilité des coeffi- 
Tableau 5. Coefficients de l'index de sélection en situation de "quota matière grasse".

\begin{tabular}{|c|c|c|c|c|c|}
\hline \multirow{3}{*}{$\begin{array}{c}\text { Situation } \\
\text { (voir tableau 1) }\end{array}$} & \multirow{3}{*}{$\begin{array}{c}\text { Marge nette } \\
\text { F/VL }\end{array}$} & \multicolumn{4}{|c|}{ Marge nette (F) / ha libéré } \\
\hline & & \multicolumn{2}{|c|}{500} & \multicolumn{2}{|c|}{1500} \\
\hline & & $\begin{array}{c}\text { Coefficient } \\
\text { MG }\end{array}$ & $\begin{array}{c}\text { Coefficient } \\
\text { TP }\end{array}$ & $\begin{array}{c}\text { Coefficient } \\
\text { MG }\end{array}$ & $\begin{array}{c}\text { Coefficient } \\
\text { TP }\end{array}$ \\
\hline $\mathrm{R}$ & $\begin{array}{l}3700 \\
4700 \\
5700\end{array}$ & $\begin{array}{r}0,07 \\
-0,04 \\
-0,14\end{array}$ & $\begin{array}{l}1,58 \\
1,62 \\
1,65\end{array}$ & $\begin{array}{r}0,13 \\
0,02 \\
-0,08\end{array}$ & $\begin{array}{l}1,61 \\
1,65 \\
1,68\end{array}$ \\
\hline B & $\begin{array}{l}2350 \\
3350\end{array}$ & $\begin{array}{r}0,10 \\
-0,06\end{array}$ & $\begin{array}{l}1,74 \\
1,79\end{array}$ & $\begin{array}{l}0,19 \\
0,03\end{array}$ & $\begin{array}{l}1,78 \\
1,83\end{array}$ \\
\hline M & $\begin{array}{l}3700 \\
4700 \\
5700\end{array}$ & $\begin{array}{r}0,03 \\
-0,07 \\
-0,18\end{array}$ & $\begin{array}{l}1,76 \\
1,80 \\
1,83\end{array}$ & $\begin{array}{r}0,10 \\
-0,01 \\
-0,12\end{array}$ & $\begin{array}{l}1,81 \\
1,84 \\
1,88\end{array}$ \\
\hline $\mathrm{H}$ & $\begin{array}{l}5800 \\
6800\end{array}$ & $\begin{array}{r}-0,10 \\
-0,19\end{array}$ & $\begin{array}{l}2,00 \\
2,03\end{array}$ & $\begin{array}{l}-0,05 \\
-0,14\end{array}$ & $\begin{array}{l}2,03 \\
2,06\end{array}$ \\
\hline $\mathrm{Ma}$ & 3700 & 0,02 & $-0,37$ & 0,08 & $-0,36$ \\
\hline & 4700 & $-0,09$ & $-0,33$ & $-0,03$ & $-0,32$ \\
\hline & 5700 & $-0,19$ & $-0,29$ & $-0,14$ & $-0,28$ \\
\hline
\end{tabular}

Le coefficient de la MP est pris égal à 1 par convention.

Tableau 6. Coefficients de l'index de sélection en situation de "quota lait".

\begin{tabular}{|c|c|c|c|c|c|}
\hline \multirow{3}{*}{$\begin{array}{c}\text { Situation } \\
\text { (voir tableau 1) }\end{array}$} & \multirow{3}{*}{$\begin{array}{c}\text { Marge nette } \\
\text { F/NL }\end{array}$} & \multicolumn{4}{|c|}{ Marge nette (F) / ha libéré } \\
\hline & & \multicolumn{2}{|c|}{500} & \multicolumn{2}{|c|}{1500} \\
\hline & & $\begin{array}{c}\text { Coefficient } \\
\text { MG }\end{array}$ & $\begin{array}{c}\text { Coefficient } \\
\text { TP }\end{array}$ & $\begin{array}{c}\text { Coefficient } \\
\text { MG }\end{array}$ & $\begin{array}{c}\text { Coefficient } \\
\text { TP }\end{array}$ \\
\hline $\mathrm{R}$ & $\begin{array}{l}3700 \\
4700 \\
5700\end{array}$ & $\begin{array}{l}0,85 \\
1,18 \\
1,87\end{array}$ & $\begin{array}{r}6,99 \\
11,18 \\
20,04\end{array}$ & $\begin{array}{l}0,71 \\
0,94 \\
1,35\end{array}$ & $\begin{array}{r}5,36 \\
8,34 \\
13,80\end{array}$ \\
\hline B & $\begin{array}{l}2350 \\
3350\end{array}$ & $\begin{array}{l}0,72 \\
1,12\end{array}$ & $\begin{array}{r}6,00 \\
11,57\end{array}$ & $\begin{array}{l}0,56 \\
0,81\end{array}$ & $\begin{array}{l}4,12 \\
7,62\end{array}$ \\
\hline M & $\begin{array}{l}3700 \\
4700 \\
5700\end{array}$ & $\begin{array}{l}0,84 \\
1,20 \\
2,02\end{array}$ & $\begin{array}{r}7,74 \\
12,67 \\
24,01\end{array}$ & $\begin{array}{l}0,69 \\
0,93 \\
1,40\end{array}$ & $\begin{array}{r}5,94 \\
9,37 \\
16,05\end{array}$ \\
\hline $\mathrm{H}$ & $\begin{array}{l}5800 \\
6800\end{array}$ & $\begin{array}{l}1,17 \\
1,77\end{array}$ & $\begin{array}{l}13,70 \\
22,71\end{array}$ & $\begin{array}{l}0,95 \\
1,33\end{array}$ & $\begin{array}{l}10,65 \\
16,48\end{array}$ \\
\hline $\mathrm{Ma}$ & $\begin{array}{l}3700 \\
4700 \\
5700\end{array}$ & $\begin{array}{l}0,80 \\
1,13 \\
1,88\end{array}$ & $\begin{array}{r}3,46 \\
6,64 \\
13,80\end{array}$ & $\begin{array}{l}0,66 \\
0,88 \\
1,32\end{array}$ & $\begin{array}{l}2,28 \\
4,50 \\
8,77\end{array}$ \\
\hline
\end{tabular}

Le coefficient de la MP est pris égal à 1 par convention.

cients en fonction des paramètres économiques considérés (tableau 6). Dans ce dernier cas, la recherche de meilleurs taux se retrouve dans la forte pondération de l'index TP et aussi dans celle de l'index MG qui favorise l'élevation du TB, du fait de la liaison génétique positive entre ces deux variables.

A titre de comparaison, l'INEL 1989 est du type MP + 0,5 MG + 3 TP. De ce fait, cet INEL convient plutôt à une situation sans quota. Il est très différent des index justifiés par le contexte "quota lait", ceux adaptés au contexte "quota matière grasse" étant intermédiaires.

\section{6 / Réponses à la sélection en situation de quota de matière grasse}

Outre l'INEL 1989, 5 index adaptés au quota MG, de la forme MP $+(0,2 ; 0,1 ; 0 ;-0,1$ ou $-0,2) \mathrm{MG}+2 \mathrm{TP}$ ont été testés pour évaluer la réponse à la sélection. Celle-ci concerne la sélection des taureaux après le testage. La pression de sélection est supposée correspondre aux $20 \%$ supérieurs pour l'index synthétique. Les taureaux sont supposés testés sur 50 filles. 
Tableau 7. Valeurs des coefficients de corrélation génétique entre caractères (Boichard et Bonaïti 1987). 1ère ligne: Holstein, 2ème ligne : races françaises.

\begin{tabular}{|c|l|l|l|l|}
\hline & MG & MP & TB & TP \\
\hline Lait & 0,6 & 0,9 & $-0,50$ & $-0,54$ \\
& 0,84 & 0,92 & $-0,3$ & $-0,43$ \\
\hline MG & & 0,73 & 0,39 & 0,05 \\
& & 0,89 & 0,26 & $-0,11$ \\
\hline MP & & & $-0,24$ & $-0,11$ \\
& & & $-0,08$ & $-0,05$ \\
\hline TB & & & & 0,67 \\
& & & & 0,59 \\
\hline
\end{tabular}

N.B. : pour le TB, $h^{2}=0,8$ en Holstein et $h^{2}=0,5$ en races françaises

Tableau 8. Réponses prédites à la sélection du 1/5 supérieur des taureaux (races Normande et Montbéliarde).

\begin{tabular}{|l|c|c|c|c|c|}
\hline Index de sélection & $\begin{array}{c}\text { Lait } \\
\mathbf{~ k g}\end{array}$ & $\begin{array}{c}\mathbf{M G} \\
\mathbf{k g}\end{array}$ & $\begin{array}{c}\mathbf{M P} \\
\mathbf{k g}\end{array}$ & $\begin{array}{c}\mathbf{T B} \\
\mathbf{g} / \mathbf{k g}\end{array}$ & $\begin{array}{c}\mathbf{T P} \\
\mathbf{g} / \mathbf{k g}\end{array}$ \\
\hline Inel 1989 & 555 & 25,3 & 19,3 & 0,51 & 0,17 \\
MP & 615 & 24,1 & 19,9 & $-0,25$ & $-0,09$ \\
$\mathrm{MG}$ & 561 & 27,1 & 17,7 & 0,82 & $-0,19$ \\
$\mathrm{MP}+0,2 \mathrm{MG}+2 \mathrm{TP}$ & 567 & 24,6 & 19,6 & 0,26 & 0,15 \\
$+0,1$ & 562 & 24,0 & 19,6 & 0,19 & 0,19 \\
+0 & 553 & 23,2 & 19,5 & 0,10 & 0,24 \\
$-0,1$ & 539 & 22,1 & 19,3 & $-0,01$ & 0,30 \\
$-0,2$ & 517 & 20,5 & 18,9 & $-0,15$ & 0,38 \\
$-0,4$ & 423 & 14,6 & 16,8 & $-0,57$ & 0,58 \\
$\mathrm{MP}+0,2 \mathrm{MG}+3 \mathrm{TP}$ & 540 & 24,1 & 19,3 & 0,40 & 0,28 \\
$+0,1$ & 530 & 23,5 & 19,2 & 0,34 & 0,34 \\
+0 & 517 & 22,6 & 19,1 & 0,27 & 0,40 \\
$-0,1$ & 497 & 21,3 & 18,8 & 0,18 & 0,48 \\
$-0,2$ & 467 & 19,5 & 18,2 & 0,07 & 0,57 \\
$-0,4$ & 355 & 13,3 & 15,6 & $-0,26$ & 0,81 \\
\hline
\end{tabular}

N.B. : pour le TB, $h^{2}=0,8$ en Holstein et $h^{2}=0,5$ en races françaises

Cinq index similaires avec un coefficient de 3 pour l'index TP ont également été envisagés car ils sont plus adaptés au règlement actuel qui n'impose pas strictement un quota MG. En effet, ce règlement est :

- moins défavorable à la MG (les valeurs économiques varient de - $1,6 \mathrm{~F}$ à $7,6 \mathrm{~F} / \mathrm{kg}$ et les coefficients de l'index $\mathrm{MG}$ sont compris entre - 0,05 et $+0,30)$.

- plus pénalisant vis à vis du vecteur (- 0,55 à $0,68 \mathrm{~F} / \mathrm{kg}$ ) et donc un peu plus incitatif à de meilleurs taux.

Les différents index testés amènent à des réponses sensiblement différentes de celles liées à l'INEL 1989. Cependant, il est nécessaire de différencier le cas des races Monbéliarde et Normande de celui de la race Holstein, en raison des différences de paramètres de variabilité génétique. En effet, en race Holstein, le taux butyreux est génétiquement plus variable que dans les races françaises et plus opposé à la quantité de lait. En conséquence, la MP (très influencée par la quantité de lait parce que le
TP est peu variable) y est plus opposée au TB que dans les races françaises. Les corrélations génétiques entre MP et TB sont de - 0,24 et de - 0,08 respectivement (tableau 7). De ce fait, les réponses du TB selon les index envisagés sont pratiquement toujours négatives en race Holstein, alors qu'au contraire, elles seront pratiquement toujours positives pour les races françaises. Il faut bien être conscient de cette singularité qui n'a rien à voir avec le raisonnement économique.

Le tableau 8 montre que dans les races françaises, les progrès génétiques sur la MP seront sensiblement les mêmes qu'avec l'INEL 1989, alors que les progrès génétiques sur la MG seront ralentis (d'environ $10 \%$ ). On pourra s'étonner que ceux-ci ne soient pas nuls, vu que le poids économique de la MG dans l'objectif de sélection est en général faible ou nul. Il faut rappeler ici que ce résultat est un résultat de génétique des populations : les liaisons génétiques entre MP et MG sont très fortes. L'augmentation de l'une entraîne une augmentation de l'autre même si cette dernière n'a pas d'intérêt économique (réponse indirecte automatique). Par ailleurs, chacun des deux taux sera amélioré avec une nette préférence pour le TP (ce qui contraste avec la réponse attendue à l'INEL 1989 où c'est le TB au contraire qui était favorisé). En effet, les progrès sur le TP seront consistants (environ doublés) alors que les progrès sur le TB seront nettement diminués (environ divisés par deux). Il y aura donc augmentation du rapport TP/TB avec une relative stabilisation $d u$ TB.

Le tableau 9 qui concerne les réponses attendues en race Holstein montre bien les spécificités liées à cette race. On y voit que l'INEL 1989 y augmente plus le TB que le TP comme dans les autres races mais que les nouveaux index envisagés entraînent des réponses négatives sur le TB et des réponses positives sur le TP, supérieures à celles obtenues avec l'INEL 1989, à condition que le coefficient du TP dans l'index synthétique soit égal à 3 . Quand ce dernier est égal à 2, la réponse sur le TP est moins bonne qu'en utilisant l'INEL 1989. Comme dans les autres races, les réponses sur la MP seront maintenues et même augmentées, alors que les réponses sur la MG seront plus freinées que dans les autres races (environ - 20\%). Les réponses différentielles selon les races ont été vérifiées sur fichiers réels, en réalisant des sélections fictives sur des séries de taureaux dont on connaît les index individuels. Le comportement spécifique de la race Holstein a ainsi été confirmé.

A titre de comparaison, on a placé au tableau concernant la Holstein les réponses attendues à une sélection selon les index officiels de différents pays (Allemagne, Italie, Pays-Bas, Grande-Bretagne, USA). On y voit que ces index sont plutôt parents avec l'INEL 1989 , avec une réponse positive très importante sur le taux de matière grasse, à l'exception des index RZW (Allemagne) et PTA \$ MFP (USA) plus laitiers mais dégradant génétiquement les deux taux. 
Tableau 9. Réponses prédites à la sélection du 1/5 supérieur des taureaux Holsteins.

\begin{tabular}{|l|c|c|c|c|c|c|}
\hline Index de sélection & $\begin{array}{c}\text { Lait } \\
\mathbf{~ k g}\end{array}$ & $\begin{array}{c}\mathbf{M G} \\
\mathbf{k g}\end{array}$ & $\begin{array}{c}\mathbf{M P} \\
\mathbf{k g}\end{array}$ & $\begin{array}{c}\text { TB } \\
\mathbf{g} / \mathbf{k g}\end{array}$ & $\begin{array}{c}\mathbf{T P} \\
\mathbf{g} / \mathbf{k g}\end{array}$ & Remarques \\
\hline INEL 1989 & 589 & 25,6 & 19,2 & 0,49 & 0,18 & \\
MP & 722 & 20,9 & 20,8 & $-0,95$ & $-0,18$ & \\
MG & 481 & 28,6 & 15,2 & 1,55 & 0,08 & \\
MP + 0,2 MG + 2 TP & 638 & 23,7 & 20,1 & $-0,06$ & 0,10 & \\
$+0,1$ & 644 & 22,6 & 20,3 & $-0,26$ & 0,10 & \\
+0 & 648 & 21,1 & 20,4 & $-0,50$ & 0,10 & \\
$-0,1$ & 648 & 19,1 & 20,4 & $-0,79$ & 0,10 & \\
$-0,2$ & 639 & 16,4 & 20,1 & $-1,14$ & 0,09 & \\
$-0,4$ & 570 & 8,0 & 17,9 & $-2,00$ & 0,08 & \\
MP + 0,2 MG + 3 TP & 603 & 23,6 & 19,8 & 0,12 & 0,21 & \\
$+0,1$ & 606 & 22,5 & 19,9 & $-0,06$ & 0,22 & \\
+ 0 & 606 & 21,0 & 20,0 & $-0,27$ & 0,23 & \\
$-0,1$ & 600 & 19,0 & 20,0 & $-0,54$ & 0,25 & \\
$-0,2$ & 586 & 16,3 & 19,6 & $-0,85$ & 0,26 & \\
$-0,4$ & 506 & 8,1 & 17,3 & $-1,64$ & 0,28 & \\
MP + 0,25 MG & 692 & 23,9 & 20,3 & $-0,33$ & $-0,12$ & RFA - RZW \\
MP + 0,09 MG - 0,015L & 474 & 22,5 & 18,3 & 0,72 & 0,59 & ITA - ILQ \\
MP + 0,17 MG - 0,013L & 474 & 24,2 & 19,2 & 0,50 & 0,35 & PB - INET \\
MP + 0,34 MG - 0,033L & 474 & 25,9 & 18,3 & 0,94 & 0,37 & BG - PIN \\
USA PTA - MFP & 757 & 23,0 & 20,0 & $-0,86$ & $-0,45$ & \\
USA Cheese yield & 602 & 20,0 & 18,0 & 0,67 & 0,00 & \\
\hline
\end{tabular}

\section{7 / Robustesse et critères de choix d'un index unique}

\section{1 / Comparaison de l'efficacité d'un index unique et d'un index adapté à chaque situation particulière}

Les résultats de la partie concernant le calcul des pondérations économiques ont bien montré qu'elles ne sont pas rigoureusement identiques d'une situation à l'autre. Par ailleurs, les réponses à la sélection dépendent des races. Dès lors, est-il raisonnable de se limiter à un seul index dans un but de simplification ? On s'est efforcé de répondre à cette question, en calculant, dans chacune des combinaisons situation $\mathrm{x}$ race, la valeur économique de la descendance de taureaux choisis d'après la combinaison d'index correspondant exactement à la situation considérée. La valeur économique de la descendance correspond alors bien entendu aux résultats des réponses à la sélection, pour chaque caractère, calculées pour cette combinaison situation $\mathrm{x}$ race et pondérées par les coefficients économiques correspondant à la situation étudiée. Par rapport à cette référence qui, par définition, correspond à l'optimum, on a chiffré en valeur relative $(\%)$, la perte de valeur économique de la descendance quand les taureaux sont choisis sur un index unique. Là aussi, les réponses par caractère ont été pondérées par les coefficients économiques correspondant à chaque situation.

Le résultat de ce calcul figure au tableau 10. On peut constater deux phénomènes :
- la perte par rapport à l'optimum liée à l'utilisation d'un index unique est en général faible, quand il s'agit des index envisagés dans la prospective. Ceci n'est en vérité pas étonnant car ils ont été choisis dans la zone qui correspondait approximativement aux pondérations théoriques données par le calcul pour les différentes situations économiques. De ce fait, l'utilisation d'un seul index national est une procédure plutôt robuste.

- l'utilisation des nouveaux index envisagés permet de corriger la situation où l'utilisation de l'INEL 1989 porte le plus de préjudice à la race Holstein, la perte d'optimalité étant environ deux fois plus importante que dans les autres races (voir tableaux 8 et 9). L'utilisation des nouveaux index en race Holstein réduit la réponse $\mathrm{MG}$ et augmente légèrement la réponse $\mathrm{MP}$, ce qui a un effet économique en moyenne favorable bien que la réponse du vecteur soit elle aussi accrue.

En conclusion, les index envisagés paraissent etre robustes en raisonnant tant entre situations qu'entre races, mais en se plaçant dans des conditions relativement futuristes de paiement du lait (fort paiement du différentiel TP et forte pénalité de la dilution des matières dans le lait). Ceci est un pari sur l'avenir qui jusqu'à présent n'a pas été concrétisé par l'industrie laitière.

Il convient de préciser, pour éviter tout malentendu, ce que signifie ici le terme "robustesse". Les résultats du tableau 3 montrent que les pondérations économiques sont moins favorables à la sélection laitière dans les situations $\mathrm{B}, \mathrm{M}, \mathrm{H}$ que dans la situation $\mathrm{R}$ (la situation 
160 / J.J. COLLEAU, D. REGALDO, P.L. GASTINEL

Tableau 10. Pertes économiques* $(\%)$ suite à la sélection sur index non optimisés en situation de quota "matière grasse".

\begin{tabular}{|c|ccccc|ccccc|}
\hline & \multicolumn{4}{|c|}{ Montbéliarde et Normande } & \multicolumn{5}{c|}{ Holstein } \\
\cline { 2 - 10 } Situation & $\mathbf{R}$ & $\mathbf{B}$ & $\mathbf{M}$ & $\mathbf{H}$ & $\mathbf{M a}$ & $\mathbf{R}$ & $\mathbf{B}$ & $\mathbf{M}$ & $\mathbf{H}$ & Ma \\
\hline Inel 1989 & 2,0 & 1,2 & 2,8 & 4,6 & 4,1 & 5,5 & 3,5 & 7,0 & 10,4 & 10,6 \\
MP + 0,2 MG + 2 TP & 0,8 & 0,4 & 1,3 & 2,7 & 2,4 & 2,3 & 1,2 & 3,4 & 5,9 & 5,7 \\
$+0,1$ & 0,4 & 0,2 & 0,8 & 1,7 & 2,2 & 1,3 & 0,6 & 2,1 & 4,1 & 4,5 \\
+0 & 0,2 & 0,2 & 0,4 & 0,9 & 2,4 & 0,6 & 0,4 & 1,1 & 2,4 & 3,5 \\
$-0,1$ & 0,5 & 0,8 & 0,3 & 0,2 & 3,1 & 0,6 & 1,1 & 0,7 & 1,2 & 3,1 \\
$-0,2$ & 1,7 & 2,4 & 1,2 & 0,3 & 4,8 & 1,9 & 3,1 & 1,5 & 0,9 & 3,9 \\
MP + 0,2 MG + 3 TP & 0,8 & 0,4 & 1,1 & 2,0 & 3,7 & 2,2 & 1,0 & 3,1 & 5,3 & 7,3 \\
$+0,1$ & 0,6 & 0,4 & 0,7 & 1,2 & 3,9 & 1,2 & 0,5 & 1,9 & 3,5 & 6,3 \\
+0 & 0,7 & 0,8 & 0,6 & 0,6 & 4,5 & 0,6 & 0,3 & 0,9 & 1,8 & 5,6 \\
$-0,1$ & 1,4 & 1,8 & 1,0 & 0,3 & 5,8 & 0,6 & 1,0 & 0,5 & 0,6 & 5,5 \\
$-0,2$ & 3,2 & 3,9 & 2,3 & 0,8 & 8,3 & 2,0 & 3,1 & 1,4 & 0,3 & 6,6 \\
\hline
\end{tabular}

* moyenne de 4 ou 6 sous situations équiprobables.

Tableau 11. Corrélations entre I'Inel 1993 et les index élémentaires, pour des taureaux nés à partir de 1985 et un coefficient de détermination de l'index supérieur ou égal à 0,5 au calcul 93/4. 1're ligne 2041 taureaux Holsteins, $2^{\text {eme }}$ ligne 410 taureaux Normands, $3^{\text {eme }}$ ligne 413 taureaux Montbéliards.

\begin{tabular}{|c|l|l|l|l|l|}
\hline & MP & MG & TP & TB & Lait \\
\hline \multirow{2}{*}{ INEL 1993 } & 0,97 & 0,7 & 0,24 & $-0,09$ & 0,72 \\
& 0,97 & 0,83 & 0,27 & $-0,04$ & 0,79 \\
& 0,98 & 0,83 & 0,18 & 0,01 & 0,82 \\
\hline MP & & 0,7 & 0,02 & $-0,24$ & 0,86 \\
& & 0,87 & 0,03 & $-0,18$ & 0,91 \\
& & 0,87 & $-0,02$ & $-0,10$ & 0,92 \\
\hline MG & & & 0,1 & 0,39 & 0,56 \\
& & & $-0,04$ & 0,18 & 0,81 \\
& & & $-0,09$ & 0,27 & 0,83 \\
\hline TP & & & & 0,65 & $-0,49$ \\
& & & & 0,58 & $-0,38$ \\
& & & & 0,56 & $-0,41$ \\
\hline TB & & & & & $-0,54$ \\
& & & & & $-0,41$ \\
& & & & & \\
\hline
\end{tabular}

Ma étant mise à part à cause de différence de système de paiement du lait), essentiellement parce que les coûts de production du lait y sont plus élevés. Les résultats du tableau 10 montrent en fait que les meilleurs taureaux sont toujours les mêmes dans toutes les situations compte tenu des informations dont on dispose à leur sujet. Seule la prise en considération d'index nouveaux pour des caractères tels que la production de viande ou la reproduction permettrait de différencier le classement optimal en fonction des conditions de milieu.

\section{2 / Choix de l'index retenu}

Il a été montré précédemment que les 10 modalités d'index envisagées ont des efficacités économiques assez voisines.

L'argument final qui a été retenu pour les départager a porté sur les capacités à stabiliser les évolutions de niveau génétique pour le taux butyreux, c'est-à-dire empêcher qu'il ne s'élève ou au contraire ne baisse trop. Un compromis raisonnable paraît être la combinaison $\mathrm{MP}+3 \mathrm{TP}$ car cette évolution est simultanément faiblement négative en Holstein et faiblement positive dans les autres races.

\section{3 / Corrélations entre le nouvel INEL et les index élémentaires}

Le tableau 11 présente d'une autre manière les propriétés de cet index, appliqué à une population de taureaux récents indexés. Il est manifeste que l'INEL 1993 ne peut être confondu avec l'index "lait" (corrélation 0,7 à 0,8 ) et que la corrélation avec l'index "taux protéique" est sensible $(0,2$ à 0,3$)$. On y observe une fois de plus la spécificité de la race Holstein. L'INEL 1993 est moins corrélé à la MG et davantage en opposition avec le TB que dans les autres races. 


\section{8 / Présentation de travaux étrangers analogues}

Le premier travail qui tienne compte de l'incidence des quotas sur les objectifs de sélection est celui de Van Arendonk et al (1985), qui est en fait une application des considérations théoriques de l'influence du redimensionnement des exploitations sur les marges économiques, considérations développées par Brascamp et al (1985) et Smith et al (1986). Dans ce travail, Van Arendonk et al envisagent deux situations extrêmes : l'une où la quantité totale de fourrages consommés sur l'exploitation ne varie pas (si les effectifs diminuent, chaque animal consomme de plus en plus de fourrage) et l'autre où, au contraire, la quantité de fourrage consommée est toujours la même, les fourrages excédentaires étant vendus (c'est une forme particulière d'activité de substitution). L'application d'un quota lait dans la première situation, considérée comme plus vraisemblable, amène à revaloriser la sélection sur les deux taux. La perte économique liée à l'application des quotas est évaluée à $18 \%$ du progrès économique annuel lié à la sélection avant l'instauration de quotas.

Gibson (1989 a) analyse par une méthodologie analogue la valeur économique du vecteur, de la matière protéique et de la matière grasse en situation de quota lait et de quota matière grasse, pour les conditions économiques anglaises. Les coûts fixes sont supposés être proportionnels au nombre de vaches. Les besoins alimentaires supplémentaires des animaux sélectionnés sont supposés être totalement couverts par le concentré. Il n'y a pas de contrainte de surface sur l'exploitation. Malgré ces hypothèses, qui peuvent être considérées comme plus sévères que les nôtres, la valeur économique de la matière grasse quand le quota correspondant est instauré ne chute que de $60 \%$. Rappelons que dans notre cas, elle est de l'ordre de $100 \%$. Le désaccord semble provenir du fait que la marge nette par vache est plus faible (3 $000 \mathrm{~F} /$ vache) dans cette étude (en tenant compte notamment des charges de travail) que dans notre cas, ce qui diminue l'impact défavorable des quotas.

Gibson (1989 b, c) analyse l'incidence de quotas pour la matière grasse dans les conditions économiques canadiennes de 1988. La méthode est analogue à celle utilisée pour l'analyse anglaise. La valeur économique de la matière grasse diminue de $58 \%$. Celle de la matière protéique reste à son niveau de départ, c'est-à-dire un niveau pratiquement nul, car seul le gramme différentiel de matière grasse était payé dans l'Ontario à l'époque. On vérifie ainsi que les conditions initiales du marché peuvent avoir une incidence prépondérante sur les résultats des simulations.

Groen (1988, 1989a, 1989b, 1990) étudie l'incidence du quota de matière grasse dans les conditions hollandaises et trouve que la valeur économique de la matière grasse diminue de $62 \%$ par rapport à la situation où il n'y a pas de quota. Dans ce modèle, l'augmentation de production par animal s'accompagne d'une éléva- tion du rapport concentré / fourrages. La réduction des temps de travail, liée au nombre plus réduit d'animaux est prise en considération. La baisse du nombre d'animaux se traduit par une baisse de la quantité de fourrages achetés. Ces deux dernières hypothèses du modèle peuvent expliquer pourquoi la diminution de valeur économique de la matière grasse est beaucoup moins drastique que dans notre cas où nous ne comptabilisons pas ces deux sources de bénéfice.

Harris et Freeman (1993) utilisent une méthode totalement différente de celle des autres auteurs. Les effets de différents niveaux possibles de progrès génétiques annuels pour un certain nombre de performances zootechniques (notamment la production de matière grasse) sont étudiés pour un troupeau initial de 80 vaches, sur une durée cumulée de 10 ans. Le bilan économique ne concerne que la partie bovine. Il n'y a aucune considération de surface. La technique dite de "programmation linéaire" est utilisée pour optimiser les rapports entre les 5 types d'aliment possibles, de manière à maximiser le revenu cumulé mais en respectant des contraintes zootechniques portant sur la composition des rations. Cependant, l'origine des sources alimentaires (ferme / extérieur) n'est pas précisée. Le poids économique des caractères à sélectionner est finalement évalué par la variation du bilan global quand le progrès génétique varie d'une unité, en maintenant les autres caractères à niveau génétique constant, et en raisonnant à effectif constant. En situation de quota pour la matière grasse, la valeur économique de la quantité de matière grasse diminue de $105 \%$, devenant ainsi légèrement négative, alors que celle de la quantité de matière protéique augmente de $25 \%$. Cette combinaison originale de résultats paraît être liée à une hypothèse essentielle non faite précédemment : la matière grasse livrée en excédent du quota n'est pas payée alors que la matière protéique liée l'est. Il s'agit donc d'une méthode d'application des quotas qui est moins sévère que celles des pénalités.

\section{9 / Discussion et conclusions}

Les raisonnements développés ici et les solutions qui en découlent doivent être considérés comme une étape dans l'ajustement des objectifs de sélection aux conditions économiques.

Certaines hypothèses, notamment les hypothèses 3 et 4 peuvent être considérées comme optimistes vis-à-vis de l'efficacité de la sélection laitière, particulièrement quand on raisonne à court terme. On peut également considérer comme optimiste le fait que la fonction économique considérée ne tienne pas compte nommément d'autres caractères zootechniques tels que l'aptitude à la reproduction ou la résistance aux maladies dont on sait qu'elles sont en association génétique défavorable avec la production laitière (Boichard 1990, Colleau et Duval 1994).

Inversement, on peut considérer comme pessimiste l'hypothèse 1 selon laquelle les quotas et surfaces par exploitation sont rigoureusement constants, même sur le long terme 
(Amon et al 1993). On peut également remarquer que les temps de travaux ne sont pas pris en compte, ce qui est pessimiste vis-à-vis des conséquences de la sélection laitière. Par ailleurs, on peut observer qu'au moins dans des conditions d'exploitation relativement intensives, la longévité est en liaison modérément positive avec le niveau de production laitière (Ducrocq et al 1988).

De fortes variations de la marge par vache ont été envisagées, ce qui devrait tempérer le reproche possible d'un centrage de la réflexion sur la zone bretonne (voir le paramétrage de la situation B qui pourrait correspondre aux zones de montagne). Dans ces conditions, il apparaît bien que la valeur économique de la MG diminue d'autant plus que la marge initiale est forte. Néanmoins, le calcul des réponses économiques à la sélection nous montre qu'il est efficace d'utiliser pratiquement les mêmes taureaux dans tous les cas. Ce résultat qui peut paraitre surprenant tient au fait que les taureaux ne sont jugés ici que sur une partie des caractères, leurs capacités laitières (au sens large), et que MG et MP sont très fortement liées. Il manque les éléments fondamentaux de jugement que sont la longévité, la reproduction et la valeur bouchère. Les pondérations optimales entre tous ces éléments dépendent alors du niveau de productivité de départ et du type de système utilisé (développement de la production viande si le niveau laitier est faible, etc).

On en arrive ainsi à la conclusion que la fonction économique à maximiser pour les troupeaux laitiers devrait être redéfinie plus finement en tenant compte des caractères autres que les composantes de la production laitière : reproduction (Boichard 1990), résistance aux mammites (Colleau et Duval 1994), longévité indépendante du niveau laitier (Beard et James 1993). Les difficultés méthodologiques de cette approche plus complète sont notablement accrues quand des contraintes de quota de production sont imposées (Van Arendonk et Brascamp 1990, Kristensen et Thysen 1991, Beard et James 1993, Harris et Freeman 1993). Ce nouvel objectif de sélection peut être optimisé à partir des seules variables de production laitière, si elles seules sont accessibles à une indexation systématique, en tenant compte des liaisons génétiques entre variables mesurées et variables non mesurées. Cependant, il est clair que des progrès génétiques intéressants sur ce nouvel objectif ne sont réalisables que si toutes les variables importantes sont sujettes à indexation. C'est le cas dans les pays scandinaves. Vu les nouvelles conditions économiques et les niveaux très importants de sélection laitière déjà obtenus, cela devrait être le cas pour les autres pays et le nôtre plus particulièrement.

Le raisonnement à court terme amène à privilégier le calcul des valeurs économiques à partir des marges brutes et non des marges nettes. Dans ce cas, pour la situation de référence $R$ avec une marge brute par vache de $9700 \mathrm{~F}$, les valeurs économiques pour le vecteur, la MG et la MP deviennent respectivement -0,48 F/kg, -11,6 F/kg, 46,3 F/kg, l'index idéal devenant MP - 0,36 MG + 1,9 TP. Pour un éleveur, un choix personnel fondé sur une telle formulation ne se justifierait que s'il n'envisage pas dans les 10 ans qui viennent, de modifications majeures de charges de structure. Par contre, on peut constater (tableaux 8 et 9) que l'utilisation de cet index dans les secteurs clefs de la sélection aurait pour effet de réduire très notablement l'accroissement des quantités de matière protéique ainsi que, le taux butyreux, notamment en race Holstein. Cela freinerait d'environ $10 \%$ la progression du bilan économique global à long terme. On peut signaler par ailleurs que l'augmentation très brutale du taux butyreux sur les vaches au contrôle laitier entre 1988 et $1992(+1,5$ $\mathrm{g} / \mathrm{kg}$ en Holstein, $+1,4 \mathrm{~g} / \mathrm{kg}$ en Montbéliarde, + $1,5 \mathrm{~g} / \mathrm{kg}$ en Normande) n'est que partiellement imputable à la génétique $(20 \%, 43 \%, 20 \%$ respectivement) comme le montrent les dépouillements détaillés (INRA - Institut de l'Elevage 1993a) : tenter de résoudre cette question par des mesures de nature uniquement génétique entraînerait des coûts à long terme.

Dans l'immédiat, la modification introduite dans la version 1993 de l'INEL est déjà substantielle, puisqu'on y estime que l'augmentation de production de matière grasse par vache n'est plus justifiée. Pour l'instant, aucun autre pays n'est allé jusque là. Limiter davantage l'accroissement de cette production avec des index où le coefficient de la MG est de - 0,2 ne se justifie économiquement que dans certains cas extrêmes de nos simulations. Par ailleurs, les conséquences à long terme d'une réduction drastique des niveaux génétiques pour le taux butyreux, ainsi encouragée, sont difficiles à appréhender, notamment pour une race internationale comme la race Holstein. Il convient de remarquer qu'indépendamment de toute redéfinition de l'INEL, les opérateurs de la sélection (Unités de sélection et éleveurs) ont déjà entamé une contre sélection vigoureuse du taux butyreux. En race Holstein, les taureaux mis au testage et nés en 1991 ont un niveau génétique sur ascendance pour le TB inférieur de $1,8 \mathrm{~g} / \mathrm{kg}$ à ceux nés en 1984 , ce qui est considérable (Gastinel, communication personnelle). Dans toutes les races, le niveau génétique de TB pour les taureaux réalisant les inséminations premières en 1992 est inférieur de 0,6 à $1 \mathrm{~g} / \mathrm{kg}$ suivant les races à celui correspondant aux inséminations de 1990 (INRA - Institut de l'Elevage 1993b). On constate que le résultat de ces choix a été de freiner l'augmentation du taux protéique. Le choix d'un coefficient 3 pour le TP dans l'INEL 1993 permet précisément d'éviter ce genre d'inconvénient.

L'utilisation du nouvel INEL amènera à augmenter au contraire le taux protéique et donc le rapport TP/TB. Dans les anciens index, ce rapport n'était pas augmenté, non pas parce qu'il était techniquement impossible de le faire mais parce que les circonstances économiques ne s'y prêtaient pas.

Pour terminer, on signalera que l'efficacité économique globale de la sélection n'est pas tota- 
lement annulée, même si elle sort amoindrie (d'environ $30 \%$ ) de l'instauration des quotas.

Les questions à long terme posées à la sélection sont d'une part l'introduction effective de nouveaux caractères qui sont de moins en moins secondaires et d'autre part, l'expression de l'ensemble des caractères génétiques, et donc leur valorisation à long terme, dans des situations où par souci de réduction des coûts, l'éleveur réduit massivement les intrants. La réponse adéquate à ce genre de questions exige non seulement un effort de modélisation mais aussi d'expérimentation. La synthèse économique des résultats techniques complets de certaines expériences de longue durée, comme celle de l'INRA au Pin-au-Haras, devrait permettre de dire si les animaux très sélectionnés sont toujours les plus efficaces en situation de conduite extensive.

\section{Remerciements}

Les auteurs remercient les trois lecteurs anonymes mandatés par la revue pour leurs commentaires et suggestions, ainsi que G. Liénard pour une lecture officieuse. 


\section{Annexe. Expression des pondérations économiques en situation de quota.}

La marge totale au niveau de l'exploitation est de la forme $M=N M_{a}+C S_{C}$

avec

$$
\begin{aligned}
& N=\text { nombre de vaches laitières } \\
& M_{a}=\text { marge par vache }=R_{a}-C_{a} \\
& R_{a}=\text { recette par vache } \\
& C_{a}=\text { coût par vache } \\
& S_{C}=\text { surface totale occupée hors atelier laitier } \\
& C=\text { valorisation de l'ha correspondant. }
\end{aligned}
$$

Les revenus et coûts bovins se rapportent à $n$ caractères zootechniques. Les performances moyennes correspondantes au niveau des animaux de l'exploitation sont notées $z_{i}(i=1$ à $n)$. La surface nécessitée par l'entretien de ces $\mathrm{N}$ vaches laitières est $\mathrm{S}=\mathrm{NS} \mathrm{S}_{\mathrm{a}}$ avec $\mathrm{S}_{\mathrm{a}}=$ surface par animal.

Les quotas qui sont imposés au niveau de l'exploitation sont de la forme $Q=N \sum_{i} q_{i} z_{i}$

$\mathrm{Si}$, par exemple, on considère 3 caractères

$$
\begin{aligned}
& 1 \text { production de matière grasse (MG) } \\
& 2 \text { production de matière protéique (MP) } \\
& 3 \text { production de vecteur }(\mathrm{V}) \text { = lait (L) - MG - MP }
\end{aligned}
$$

alors le quota matière grasse se traduit par $q_{1}=1, q_{2}=0, q_{3}=0$

le quota lait se traduit par $q_{1}=q_{2}=q_{3}=1$

S'il y a un quota lait avec pénalité pour le taux butyreux (TB)

quand il dépasse un taux de référence $\left(\mathrm{TB}_{0}\right)$, on a alors

avec $\quad k=$ coefficient constant

On en déduit $\frac{Q}{-}=\left(1-k T B_{0}+k\right) M G+\left(1-k B_{0}\right) M P+\left(1-k T B_{0}\right) V$

$\mathrm{N}$

Si $k=17,5$ pour des taux exprimés en fractions $\left(\mathrm{TB}_{0}=0,038\right.$ par exemple)

$$
\mathrm{q}_{1}=17,83 \quad \mathrm{q}_{2}=\mathrm{q}_{3}=0,33
$$

Le poids économique $\mathrm{m}_{\mathrm{i}}$ du caractère $\mathrm{z}_{\mathrm{i}}$ correspond à la variation de marge pour l'exploitation lorsque $\mathrm{z}_{\mathrm{i}}$ varie d'une unité, en raisonnant au niveau de l'animal, c'est-à-dire :

$\mathrm{m}_{\mathrm{i}}=\frac{1}{\mathrm{~N}} \frac{\delta \mathrm{M}}{\delta \mathrm{z}_{\mathrm{i}}} \quad$ (1) où $\frac{\delta \mathrm{M}}{\delta \mathrm{z}_{\mathrm{i}}}$ désigne la dérivée de $\mathrm{M}$ par rapport à $\mathrm{z}_{\mathrm{i}}$

On $\mathrm{a} \frac{\delta \mathrm{M}}{\delta \mathrm{z}_{\mathrm{i}}}=\frac{\delta \mathrm{N}}{\delta \mathrm{z}_{\mathrm{i}}} \mathrm{M}_{\mathrm{a}}+\mathrm{N} \frac{\delta \mathrm{M}_{\mathrm{a}}}{\delta \mathrm{z}_{\mathrm{i}}}+\mathrm{c} \frac{\delta \mathrm{S}_{\mathrm{c}}}{\delta \mathrm{z}_{\mathrm{i}}}$

La contrainte des quotas se traduit par:

$$
0=\frac{\delta N}{\delta z_{i}} \sum q_{i} z_{i}+N q_{i}
$$

La contrainte de surface totale $\left(S+S_{c}\right)$ constante se traduit par : $\quad 0=\frac{\delta N}{\delta z_{i}} S_{a}+N \frac{\delta S_{a}}{\delta z_{i}}+\frac{\delta S_{c}}{\delta z_{i}}$

$$
\text { De (3) et (4), on déduit } \frac{\delta \mathrm{N}}{\delta \mathrm{z}_{\mathrm{i}}}=-\mathrm{N} \frac{\mathrm{q}_{\mathrm{i}}}{\sum \mathrm{q}_{\mathrm{i}} \mathrm{z}_{\mathrm{i}}} \quad \text { (5) } \quad \frac{\delta \mathrm{S}_{\mathrm{c}}}{\delta \mathrm{z}_{\mathrm{i}}}=\mathrm{N} \frac{\mathrm{q}_{\mathrm{i}} \mathrm{s}_{\mathrm{a}}}{\sum \mathrm{q}_{\mathrm{i}}}-\mathrm{N} \frac{\delta \mathrm{S}_{\mathrm{a}}}{\delta \mathrm{z}_{\mathrm{i}}}
$$

On trouve alors, à partir de (1), (2), (5) et (6) :

$$
\mathrm{m}_{\mathrm{i}}=\left[\frac{\delta \mathrm{M}_{\mathrm{a}}}{\delta \mathrm{z}_{\mathrm{i}}}-\mathrm{c} \frac{\delta \mathrm{S}_{\mathrm{a}}}{\delta \mathrm{z}_{\mathrm{i}}}\right]-\frac{\mathrm{q}_{\mathrm{i}}}{\sum \mathrm{q}_{\mathrm{i}} \mathrm{z}_{\mathrm{i}}}\left(\mathrm{M}_{\mathrm{a}}-\mathrm{cS}_{\mathrm{a}}\right)
$$

(a) (b) $\quad$ (c)

- Le terme (a) correspond à la variation de marge par animal quand $z_{i}$ augmente de 1 (marge classique).

- Le terme (b) correspond à la variation de marge liée à l'activité hors atelier laitier,

à effectifs bovins constants, quand $z_{\mathrm{i}}$ augmente de 1 .

- Le terme (c) correspond à la variation de marge due à la variation d'effectif liée au respect de la contrainte de quota $=$ variation d'effectif $\left(\mathrm{q}_{\mathrm{i}} / \Sigma \mathrm{q}_{\mathrm{i}} \mathrm{z}_{\mathrm{i}}\right)$ multipliée par le bénéfice résultant de l'activité bovine plutôt que des activités de substitution $\left(\mathrm{M}_{\mathrm{a}}-\mathrm{c} \mathrm{S}_{\mathrm{a}}\right)$. 
Un exemple numérique en situation de quota matière grasse.

- Hypothèses

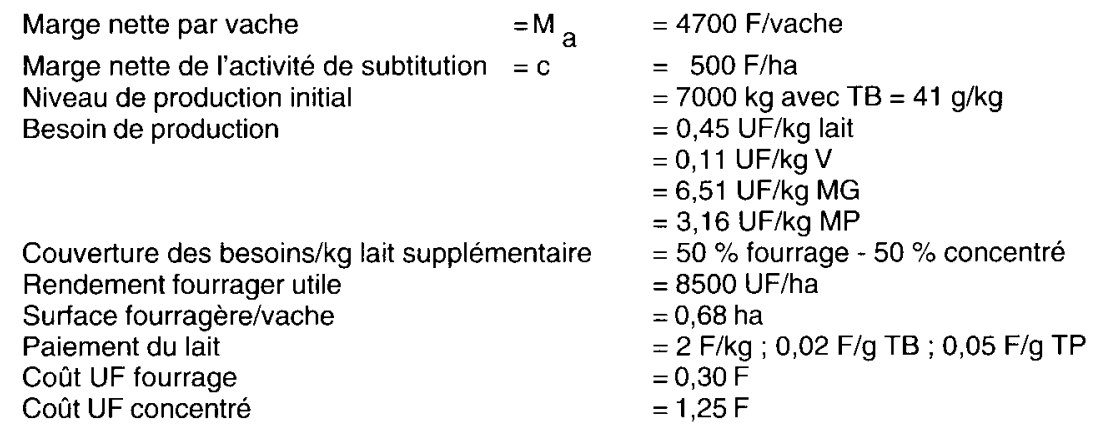

- Calcul du terme (a)

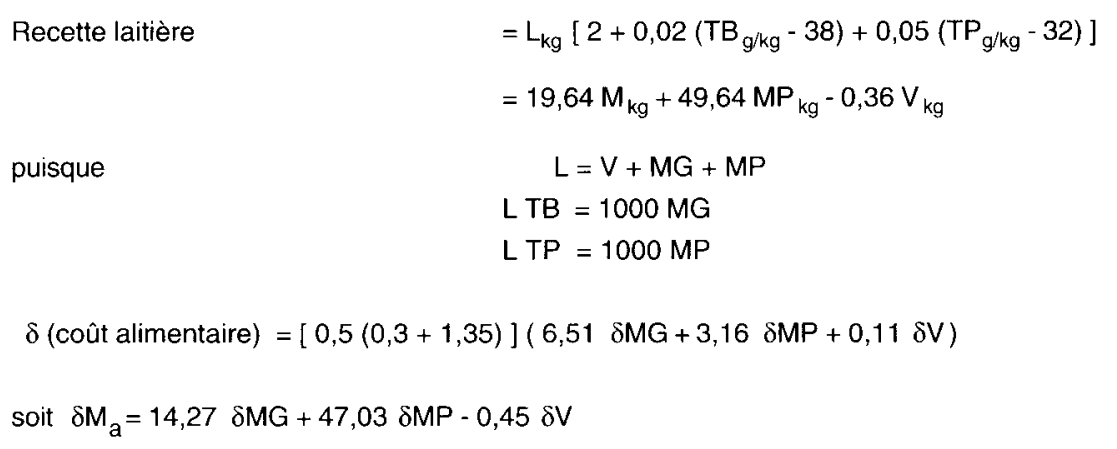

- Calcul du terme (b)

$$
\begin{aligned}
-\mathrm{c} \delta \mathrm{Sa} & =-500 \frac{0,5}{8500}(6,51 \delta \mathrm{MG}+3,16 \delta \mathrm{MP}+0,11 \delta \mathrm{V}) \\
& =-0,19 \delta \mathrm{MG}-0,09 \delta \mathrm{MP}-\varepsilon \delta \mathrm{V}
\end{aligned}
$$

- Calcul du terme (c)

$$
\begin{aligned}
& \text { pour MG }(i=1) \\
& -\frac{1}{7000 \times 0,041}(4700-500 \times 0,68)=-15,26 \mathrm{~F}
\end{aligned}
$$

pour MP ou V ( $i=2$ ou 3$)$

0

D'où le résultat

\section{Valeur économique}

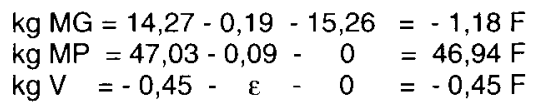




\section{Références bibliographiques}

Amon G., Liénard G., Delattre J.C., Heroguelle E., 1993. Production laitière intensive dans le Pas-deCalais. Fonctionnement, adaptation aux quotas et résultats techniques et économiques de 22 exploitations sur 6 campagnes. INRA Prod. Anim., 6, 117-136.

Anonyme, 1987. L'expérience et le savoir-faire des Eleveurs en Bretagne et Pays de Loire. 9 cas-types (EBD - ITEB - Chambres d'Agriculture-EDE Bretagne et Pays de Loire).

Anonyme, 1991. Produire du lait et des taurillons dans l'Est. 10 savoir-faire mis au point chez des éleveurs de l'Est de la France. (Chambre d'Agriculture - EDE - SUAD de l'Est - EBD - ITEB).

Beard K.T., James J.W., 1993. Including the involuntary component of longevity in a breeding objective. 44th Annual Meeting of the EAAP, Aarhus, Denmark.

Boichard D., 1990. Estimation of the economic value of conception rate in dairy cattle. Livest. Prod. Sci, $24,187-204$.

Boichard D., Bonaiti M., 1987. Genetic parameters for first lactation dairy traits in Friesian, Monthéliarde and Normande breeds. Génét. Sél. Evol., 19, 337-350.

Brascamp E.W., Smith C., Guy D.R. 1985. Derivation of economic weights from profit equations. Anim. Prod., 40, 175-180.

Colleau J.J., Duval E., 1994. A simulation study on selection methods for improving mestitis resistance in dairy cattle. J. Dairy Sci., soumis pour publication.

Ducrocq V., Quaas R.L., Pollak E.J., Casella G., 1988. Length of productive life of dairy cows. 2 variance component estimation and sire evaluation. J. Dairy Sci, 71, 3071-3085.

Elsen J.M., Bibé B., Landais E., Ricordeau G. 1986. Twenty remarks on economic evaluation of selection goals. 3rd World congress on genetics applied to livestock production. Dickerson G.E. Johnson R.K. editors. University of Nebraska, XII, 321-327.

Gibson J.P., 1989a. Economic weights and index selection of milk production traits when multiple production quota apply. Anim. Prod., 49, 171-181.

Gibson J.P., 1989b. Selection on the major components of milk : alternative methods of deriving economic weights. J. Dairy. Sci., 72, 3176-3189.

Gibson J.P., 1989c. The effect of pricing systems, economic weights, and population parameters on economic response to selection on milk components. J. Dairy, Sci., 72, 3314-3326.

Groen A.F. 1988. Derivation of economic values in cattle breeding. A model at farm level. Agric. Sys., $27,195-213$.
Groen A.F., 1989a. Economic values in cattle breeding I. Influences of production circumstances in situations without output limitations. Livest. Prod. Sci., 22, 1-16.

Groen, A.F., 1989b. Economic values in cattle breeding. II. Influences of production circumstances in situations with output limitations. Livest. Prod. Sci., 22, 17-30.

Groen A.F., 1990. Influences of production circumstances on the economic revenue of cattle breeding programmes. Anim. Prod., 51, 469-480.

Harris B.L., Freeman A.E., 1993. Economic weights for milk yield traits and herd life under various economic conditions and production quotas. J. Dairy. Sci., 76, 868-879.

Hazel L.N., 1943. The genetic basis for construction of selection indexes. Genetics., 28, 476-490.

INRA-Institut de l'Elevage, 1993a. Le cheptel des trois principales races laitières françaises. Evolution génétique et phénotypique 1982-1992. Prévision d'évolution génétique 1993-1998. INRA - Institut de l'Elevage $\mathrm{n}^{\circ} 2129,50 \mathrm{pp}$.

INRA-Institut de l'Elevage, 1993b. Bilan génétique de l'insémination artificielle en races laitières bovines. Résultats 1992 . Compte-rendu INRA Institut de l'élevage ${ }^{\circ} 2096,65 p p$.

Kristensen R.A., Thysen I. 1991. Economic value of culling information in the presence or absence of milk quota. Acta. Agric. Scand., 41, 129-135.

Minvielle F., 1990. Principes d'amélioration génétique des animaux domestiques. INRA Paris. $211 \mathrm{pp}$.

Ollivier L., 1981. Eléments de génétique quantitative. Masson Paris. 152pp.

Smith C., James J.W., Brascamp E.W. 1986. On the derivation of economic weights in livestock improvement. Anim. Prod., 43, 545-551.

Van Arendonk J.A.M., Wilmink J.B.M., Dijkhuizen A.A., 1985. Consequences of a restriction of the herd milk production for the selection on milk, fat and protein. Adapting EEC cattle breeding programmes to market realities. Krausslich $\mathrm{H}$., and $\mathrm{A}$. Lutterback, Editors. Commission of the European Communities, Brussels, pp 211-220.

Van Arendonk J.A.M., Brascamp E.W., 1990. Economic consideration in dairy cattle breeding. 4th World Congress on genetics applied to livestock production. Thompson R, Woolliams J.A., Editors. Organizing Committee, Edinburgh. , XIV, pp 78-85.

Wilmink J.B.M., 1988. Selection on fat and protein to maximise profit in dairy herds. Livest. Prod. Sci. 20, 299-316. 


\section{Summary}

Adjusting the French dairy selection index (INEL) to a quota situation

Selection of dairy cattle in France is carried out by means of an overall economic index for dairy traits (INEL). Weights for each trait were updated in 1993 to account for the occurrence of milk quotas and additional penalty when fat content is higher than a reference.

The paper clarifies which rationale, assumptions and parameters were used, from both economic and genetic viewpoints, to examine potential of alternative solutions. Most important assumptions were that quotas and surfaces were constant over time and that economic margins accounted for fixed costs.

The eventual solution (INEL 1993) gave no weight to fat yield per cow since INEL $1993=1.15$ (protein yield $(\mathrm{kg})+3$ protein content $(\mathrm{g} / \mathrm{kg}))$. Selection according this index is expected to increase protein content and protein/fat ratio. Fat content is expected to decrease for the Holstein breed and to be almost constant for the Normande and Montbéliarde breeds .

COLLEAU J.J., REGALDO D., GASTINEL P.L., 1994. Adaptation de l'index francais de sélection laitière (INEL) au contexte des quotas. INRA Prod. Anim., 7 (3), 151-167. 